\title{
Targeting Posttranslational Modifications - Perspectives for Biocatalyst Engineering
}

\author{
Beate Pscheidt ${ }^{\mathrm{a}}$, Roland Weis ${ }^{\mathrm{a}}$, and Anton Glieder ${ }^{\mathrm{a}, \mathrm{b}^{\star}}$
}

\begin{abstract}
Engineering posttranslational modifications is of great importance in biomedical research. Recent advances in the field of biocatalyst engineering expanded the strategy of targeting posttranslational modifications to a novel research field, i.e. rendering the particular enzyme more efficient for the desired biocatalytic conversion. Thus enzymes could be trimmed to higher fitness for technical applications e.g. by improved activity and/or prolonged life-time. Furthermore, bottlenecks during enzyme expression caused by posttranslational processing in the cell can be overcome. Focusing especially on recent approaches in our laboratory, this article presents strategies for targeted manipulations of selected posttranslational modifications.
\end{abstract}

Keywords: Biocatalysis · Hydroxynitrile lyase (HNL) · N-Glycosylation · Pichia pastoris ·

Posttranslational modification

\begin{abstract}
Abbreviations
ER: Endoplasmatic Reticulum

GalNAc: N-Acetylgalactosamin

GlcNAc: N-Acetylglucosamin

Man: Mannose

Glc: Glucose
\end{abstract}

PTM: Posttranslational modification

PaHNL5: Prunus amygdalus hydroxynitrile lyase isoenzyme 5

PEG: polyethylene glycol

\section{Introduction}

The majority of the early work on heterologous expression of recombinant proteins was based on prokaryotic hosts, mainly Escherichia coli [1]. The ease of genetic manipulation, the possibility of large-scale

\footnotetext{
${ }^{\star}$ Correspondence: Prof. A. Glieder ${ }^{a, b}$

aResearch Centre Applied Biocatalysis

Petersgasse 14, 8010 Graz, Austria

Tel: +43 3168734074

Fax: +43316 8739302

E-Mail: glieder@glieder.com

bInstitute of Molecular Biotechnology, Graz University

of Technology, Austria
}

fermentation, rapid growth, and high expression levels of recombinant protein were the main advantages in using prokaryotes as production systems. However, several posttranslational modifications (PTMs) including pro-peptide processing, signal peptide cleavage, disulfide bond formation, correct protein folding, and glycosylation of eukaryotic proteins cannot be carried out correctly in prokaryotic hosts. While proteins that do not require extensive posttranslational processing for biological activity, such as insulin, can be produced in microbial expression systems, complex proteins are not always properly processed in prokaryotes and may lack the desired biological activity [2]. In view of the limitations of prokaryotic hosts, efficient eukaryotic expression systems such as yeast, fungi, insect cells, plants, mammalian cell cultures, and transgenic animals were established. However, these production systems suffer from one or more of the following disadvantages: high production costs, difficulties in scaling up to large volumes, secretion problems, low product yields or potential contamination by viruses or prions [1]. Moreover, inappropriate PTMs can lead to negative immunogenic responses and diseases e.g. hemophilia A, the oldest known hereditary bleeding disorder where abnormal N-glycosylation blocks the procoagulant activity of factor VIII, a blood clotting protein [3].

In order to get rid of disadvantageous, inappropriate PTMs several attempts were recently made to modify different eukaryotic hosts. A strategy using the co-expression of the protease PACE/furin with the human Protein $\mathrm{C}$ in cells of mammary gland led to an increased amount of properly proteolytically processed Protein C [4]. Hollister et al. [5] succeeded in engineering a protein $\mathrm{N}$-glycosylation pathway in insect cells enabling the use of insect cells as hosts for baculovirus-mediated recombinant glycoprotein production. Transgenic insect cell lines with mammalian glycosyltransferases were isolated which could be used to produce terminally galactosylated or sialylated N-glycans. Advances in the control of PTMs in transgenic plants and their capability to perform (to some extent) humanlike modifications of recombinant proteins were also reported [1]. Even cell-free protein synthesis methods coupled with cellfree posttranslational modifications for the production of valuable proteins were developed [6]. In addition, humanization of the glycosylation pathway in the yeast Pichia pastoris leading to a human glycoprotein secreted with uniform complex N-glycosylation was achieved [2]. In the course of this work, a Pichia pastoris strain was engineered by eliminating endogenous yeast glycosylation pathways and generating a synthetic in vivo glycosylation pathway by recombinantly expressing five active eukaryotic proteins (mannosidases I and II, N-acetylglucosaminyltransferases I and II and uridine 5'-diphosphate (UDP)-Nacetylglucosamine transporter). The ability to generate human glycoproteins with homogenous glycan structures was a promising step towards producing therapeutic glycoproteins in yeasts [2]. 
In the field of therapeutic proteins numerous studies have been carried out to improve the posttranslational processing of proteins. However, the targeting of posttranslational modifications could also provide useful consequences for industrial enzymes used in biocatalysis. In this review, we focus on specific posttranslational modifications which can be used as targets for possible improvements of industrial enzymes. Especially recent reports on changed PTM patterns of highly active muteins of the $(R)$-hydroxynitrile lyase isoenzyme 5 (PaHNL5) from Prunus amygdalus which were recombinantly expressed in Pichia pastoris are highlighted.

\section{In vivo and in vitro \\ Posttranslational Modifications and their Effects on Proteins}

Inappropriate posttranslational modifications often result in improperly folded proteins lacking catalytic activity. Wellknown modifications include: $\mathrm{N}$ - and $\mathrm{O}-$ glycosylation, phosphorylation, methylation, ADP-ribosylation, proteolytic processing, sulfation, prenylation, farnesylation, acylation (myristoylation, palmitoylation), glycosylphosphatidylinositol (GPI) anchor formation, hydroxylation, acetylation, and deamidation. All of these are performed in different compartments of the cell, whereas PEGylation represents one of the in vitro manipulation methods attaching polyethylene glycol chains to proteins.

In the following, we shortly describe particular posttranslational modifications which were found to influence catalytic activity or process properties, thereby representing interesting targets for biocatalyst engineering.

\subsection{Glycosylation}

Glycosylation is regarded to be one of the most important posttranslational modifications of synthesized proteins [7]. Glycosylation represents the covalent linkage of an oligosaccharide moiety to a specific amino acid residue of a protein.

O-glycans are most frequently linked to serine (Ser) or threonine (Thr) residues and contain either just one sugar (i.e. fucose, glucose or GalNAc) or several sugars [1]. For example, mucin-like oligosaccharides can be attached to the core GalNAc sugars linked to the Ser or Thr residue. O-glycosylation is a stepwise process taking place in the Golgi apparatus where glycosyltranferases catalyze the transfer of sugar moieties from nucleotide-sugar donors onto the growing O-glycan.

$\mathrm{N}$-glycosylation in eukaryotic cells is a co-translational event where a growing polypeptide chain is decorated with a preformed oligosaccharide precursor

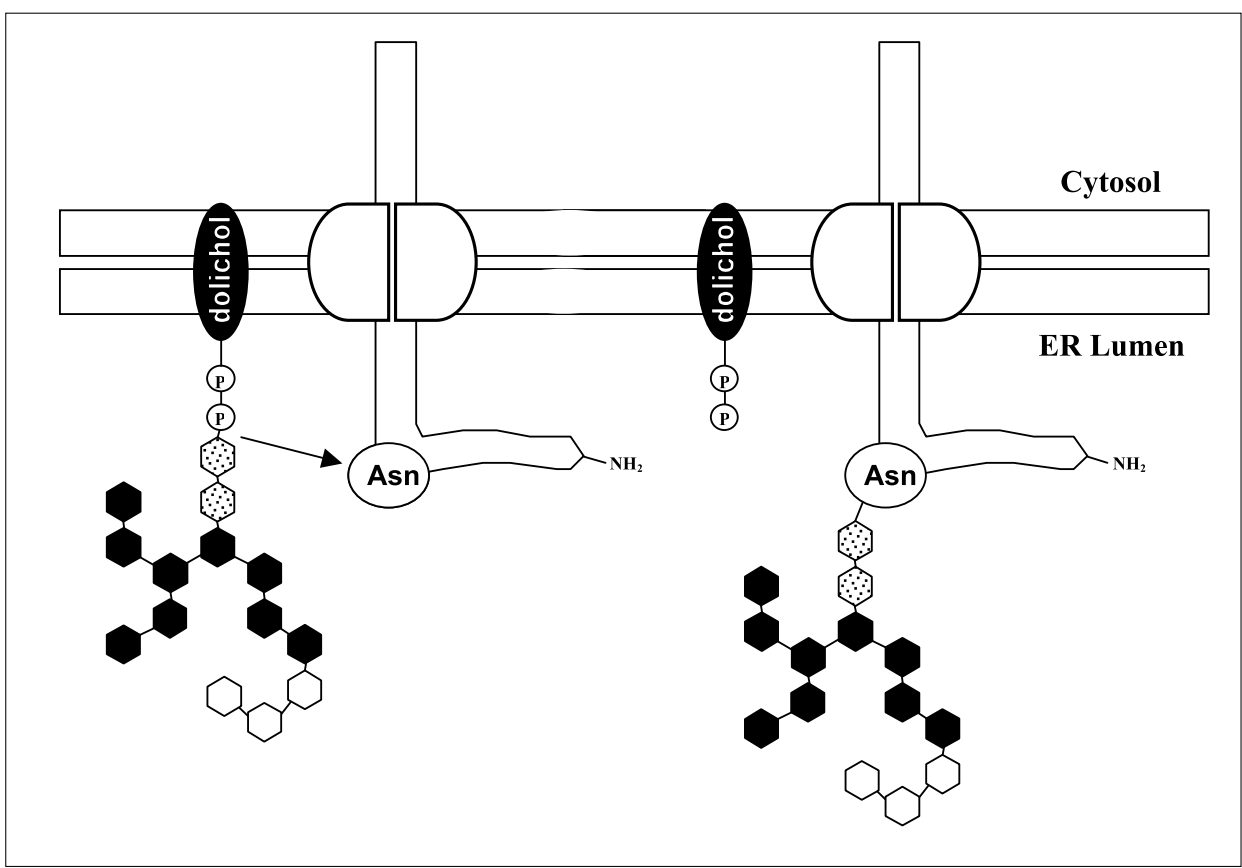

Fig. 1. N-Glycosylation in eukaryotic cells: Almost as soon as the growing polypeptide chain enters the ER it is decorated with a preformed oligosaccharide precursor $\left(\mathrm{Glc}_{3} \mathrm{Man}_{9} \mathrm{GlcNAc}_{2}\right)$ on a specific asparagine (Asn) residue (dotted hexagon: GlcNAc, black hexagon: Man, white hexagon: Glc, circle: phosphate).

$\left(\mathrm{Glc}_{3} \mathrm{Man}_{9} \mathrm{GlcNAc}_{2}\right)$ as soon as it enters the ER [8] (Fig. 1). The acceptor of the activated core-oligosaccharide is a specific asparagine (Asn) residue which is part of the potential N-glycosylation-specific motif (Asn-Xxx-Thr/Ser-Yyy). Proline (Pro) at positions $\mathrm{Xxx}$ and Yyy prohibits the formation of $\mathrm{N}$-glycosylation at this position [9][10].

Several effects of N-glycosylation were studied: O'Connor and Imperiali [11] reported that $\mathrm{N}$-glycosylation influences the conformational dynamics of the nascent polypeptide chain, hence controlling protein folding. Furthermore, the oligosaccharide chains act as shield around the peptide increasing thermal stability [12][13], solubility [14], overall rigidity [15], and resistance to protease digestion [16]. In addition, attached oligosaccharides can influence the specific activity of enzymes and lead to a rise in stability at acidic or basic $\mathrm{pH}$-values [17]. In general, attached sugar moieties stabilize the conformation of the mature glycoprotein [13][18].

\subsection{Sulfation}

Sulfation is a key posttranslational modification influencing cell-cell and cell-matrix communication [19]. Sulfation involves the attachment of a sulfate moiety to a hydroxyl or, less frequently, an amino group on a biomolecule. Most thoroughly investigated is tyrosine $\mathrm{O}$-sulfation catalyzed by tyrosylprotein sulfotransferases (TPST), which represent enzymes that transfer sulfate from the universal sulfate-donor adenosine 3'-phosphate 5'-phosphosulfate to the hydroxyl group of a protein's tyrosine residue to form a tyrosine $\mathrm{O}^{4}$-sulfate ester and 3',5'-ADP. In addition, it was reported that tyrosine O-sulfation occurred in the transGolgi network, where the enzyme was proposed to be membrane-bound with its active site oriented towards the lumen in highly enriched Golgi membranes [20-22]. Thus, $\mathrm{O}$-sulfation only takes place if the protein is shuttled through the trans-Golgi network. Moore [23] reviewed the role of tyrosine Osulfation in protein function and concluded that for the majority of tyrosine-sulfated proteins no distinct role concerning protein function has been described. Still, in some cases sulfation plays an important role in protein-protein interactions: In comparison to the modified unsulfated hirudin, the native sulfated form showed a 10-fold higher affinity for thrombin [24]. One piece of evidence for negative consequences of lacking tyrosine O-sulfation in vivo is known from the cases of mild to moderate hemophilia A which are caused by missense mutations in the factor VIII gene i.e. substitution of phenylalanine (Phe) for tyrosine (Tyr). The sulfation of Tyr1680 in factor VIII is necessary for binding to von Willebrand factor, which has the role of a carrier protein for factor VIII in the plasma causing a rise in circulation half-life of factor VIII in vivo [25][26]. Furthermore, Moore [23] reported on male mice with deficient fertility upon disruption of the Tps 1 and Tpst 2 genes.

Although the role of sulfation is being increasingly elucidated in the field of physiology and pathology, no attempts were made so far to target sulfation sites 
of biocatalysts. Beinfeld [27] proposed that sulfated tyrosines may have an impact on the solubility and stability of the Cholecystokinin (CCK) peptides, which represent gastrointestinal hormonal substances regulating pancreatic enzyme secretion and gall bladder contraction. The trial to prevent the sulfation through treatment of CCK expressing tumor cells with sodium chlorate caused a large decrease in produced CCK.

Regarding the biological purpose of sulfated glycoproteins and proteoglycans which are responsible for extracellular cell-cell and cell-matrix interactions, one could think of introducing sulfation sites and thereby providing interesting targets for future protein adhesion strategies.

\subsection{Deamidation}

As a non-enzymatic modification of proteins, deamidation is reported to be a common PTM of therapeutic proteins produced in animal expression systems that can adversely affect the structure and function of a protein rendering it more susceptible to proteolysis [1]. While it is regarded to be responsible for the decreased biological activity of some recombinant proteins like human deoxyribonuclease 1 [28], other recombinant proteins such as human growth hormone did not seem to be negatively affected by deamidation [29]. The molecular mechanism proposed for deamidation reactions of Asn residues includes the formation of a cyclic succinimidyl intermediate from whose opening aspartyl and isoaspartyl residues are formed [30]. Comparison of amino acid sequences revealed that Asn residues undergo deamidation preferably when they are located next to small, non-bulky side chains such as glycine (Gly), Ser, or alanine (Ala). These residues can be brought closely to the amide residue by the tertiary structure of the protein as well [31]. Especially for the spontaneous deamidation of glutamine (Gln) residues, the surrounding was proven to be an important determinant [31-33]. Posttranslational deamidation has multiple effects. Hemoglobin J Sardegna, a human $\mathrm{Hb}$ variant which undergoes a posttranslational deamidation process, transforming an Asn to an aspartate (Asp) residue, is one of the most common $\alpha$-globin structural mutants and is reported to be associated with thalassemic diseases [34]. Moreover, deamidation can affect the thermostability of enzymes [35-37] and lead to irreversible inactivation of $B$. licheniformis' $\alpha$-amylase [38][39]. Oh et al. [37] proposed that as a consequence of deamidation, negatively charged amino acid residues are crowded, causing a destabilization of the protein's native structure. Still, deamidation of Asn as well as Gln residues seems to make up a rather underestimated event in the field of biocatalyst engineering. Recently, Gaisberger et al. [40] reported on anticipation of the posttranslational deamidation of an Asn residue in a biocatalytically useful mutein of Prunus amygdalus hydroxynitrile lyase isoenzyme 5 ( $\mathrm{PaHNL}$ ), thereby removing a bottleneck in enzyme productivity.

\subsection{PEGylation}

An effective in vitro posttranslational modification is PEGylation. It was a challenge for years to overcome the disadvantages related to polypeptide drugs. Shortcomings limiting their usefulness include their low solubility, high susceptibility to proteolytic degradation, short circulation half-life, short shelf life and their tendency to generate neutralizing antibodies. PEGylation was reported to provide a technology to overcome these deficiencies [41][42].

The formation of PEG includes a process where repeating units of ethylene glycol are linked to form polymers with linear or branched shapes of different molecular masses. After activating a PEG polymer with a functional group - such as active carbonate, active ester, aldehyde or tresylate - it can be coupled to reactive sites on the target molecule. The most common reactive sites on polypeptides are the $\alpha$ or $\varepsilon$ amino groups of lysine, $\mathrm{N}$-terminal amino acid groups of other amino acids [43] or thiol groups of cystein residues which can be added precisely at the desired site by genetic engineering [44]. The chemical attachment of PEG to these sites is called PEGylation.

In solution, each ethylene glycol subunit of PEG is tightly associated with water molecules which trigger the PEGylated compound to behave as if it were five to ten times larger than a corresponding soluble protein of similar molecular mass. This was confirmed through size-exclusion chromatography and gel electrophoresis [45]. The PEG polymer, together with the associated water, acts as a shield protecting the attached protein from enzymatic degradation, rapid renal clearance or interactions with cell surface proteins, hence limiting adverse immunological effects. PEGylation methods also focus on the creation of larger PEG polymers to improve the pharmacokinetic and pharmacodynamic effects, since larger molecules are cleared more slowly by the kidney. Furthermore, it was reported that PEGylated drugs were more stable over a wide range of $\mathrm{pH}$ and temperature compared to their unPEGylated counterparts [46]. Harris and Chess [41] reviewed a variety of PEGylated drugs which are already in use. To mention only a few of them, a PEGylated form of adenosine deaminase can be used for the treatment of severe combined immunodeficiency disease (SCID), a PEGylated asparaginase for the treatment of leukaemia, and PEGylation of interferon $\alpha$ resulted in a more effective drug against hepatitis C. Moreover, Inada et al. [47] re- ported on the application of PEGylated enzymes for biotransformations. PEGylated lipases showed an increased thermostability at $55{ }^{\circ} \mathrm{C}$ and a $30 \%$ higher activity in aqueous media.

\section{Case Study: Targeting Posttranslational Modification of PaHNL5}

\subsection{N-Glycosylation}

Due to its $13 \mathrm{~N}$-glycosylation sites, Prunus amygdalus hydroxynitrile lyase isoenzyme 5 (PaHNL5) was secreted in a massively glycosylated form by its recombinant host Pichia pastoris [48]. In contrast to enzyme preparations from natural sources, the recombinantly produced $\mathrm{PaHNL} 5$ exhibited high stability in a broad $\mathrm{pH}$ range from 2.5 to $>6.5$. This enzyme property provides important advantages for stereoselectivity of the catalyzed reaction, i.e. the synthesis of enantiopure cyanohydrins through stereospecific enzymatic addition of $\mathrm{HCN}$ to aldehydes and ketones [49][50]. The resulting enantiopure cyanohydrins represent important intermediates for the syntheses of chiral building blocks for pharmaceuticals or agrochemicals.

At low $\mathrm{pH}$, the produced cyanohydrins are stable and the competitive chemical reaction which leads to racemic products is suppressed. Suspecting the massive Nglycosylation of PaHNL5 to be at least in part responsible for the extraordinary stability at low $\mathrm{pH}$, Weis et al. [51] reported on the use of serine scanning as a method to elucidate the influence of the N-glycosylation sites on this specific property. All asparagine residues which are included in N-glycosylation specific motifs in the $P a \mathrm{HNL} 5$ gene were individually changed to serine coding triplets. The corresponding 13 glyco-muteins were well expressed and secreted, and their specific activities for the hydrolysis of the natural substrate mandelonitrile (benzaldehyde cyanohydrin) were analyzed. A standardized photometric assay tracking the increase in absorption of benzaldehyde at $280 \mathrm{~nm}$ was employed [52]. By normalizing the calculated activity with the corresponding protein concentrations, the specific activities for all tested enzyme variants were obtained and compared. The individual stability of these variants at low $\mathrm{pH}$-values was determined by measuring the decrease in catalytic activity as a function of time. Interestingly, the results indicated that the glycosylation at position N118 seemed to be essential for stability at low $\mathrm{pH}$. The mutant N118S showed almost linear inactivation behaviour at $\mathrm{pH} 3$. While the activity after incubation in standard $\mathrm{pH}$ was retained, less than $60 \%$ activity was left after $5 \mathrm{~min}$ and only $24 \%$ activity after 10 min, respectively (Fig. 2). To prove these 


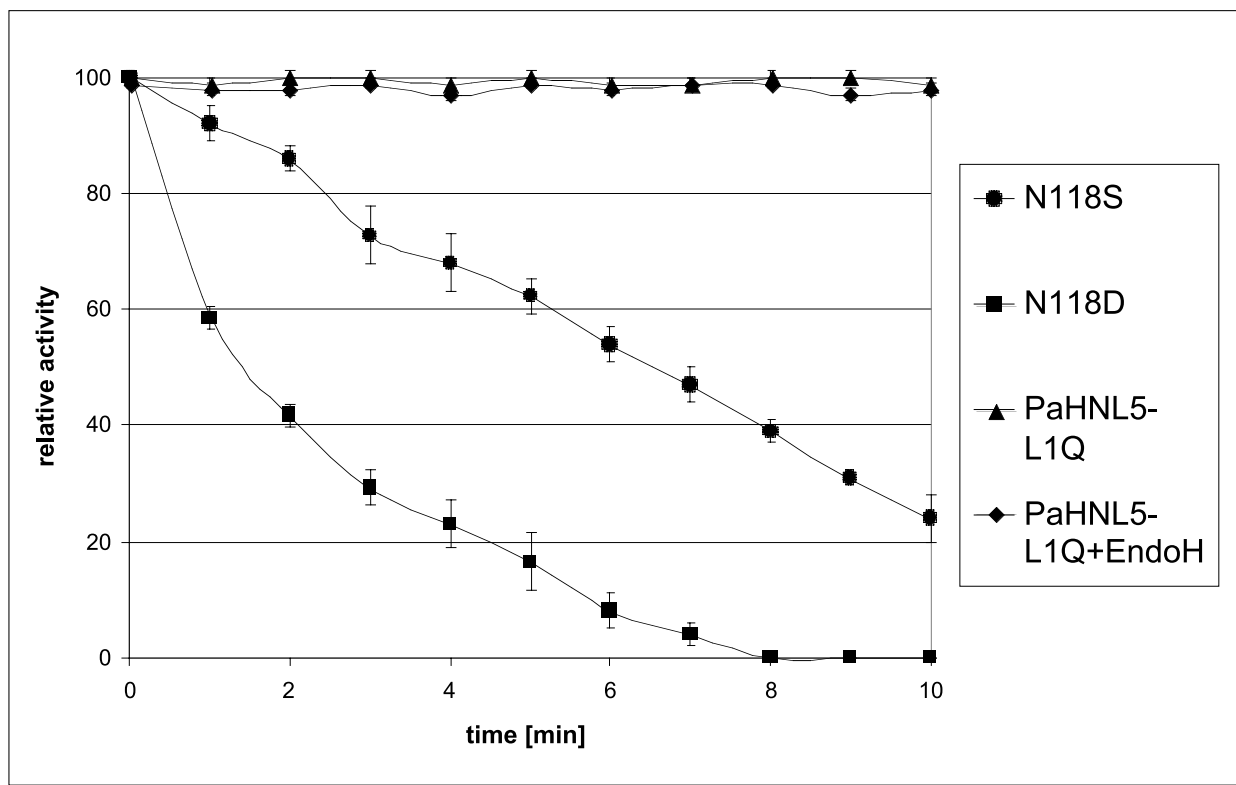

Fig. 2. Relative activities of PaHNL5-L1Q, the EndoH-deglycosylated PaHNL5-L1Q and N118 glycovariants of $P a H N L 5-L 1 Q$ using mandelonitrile as a substrate after varying incubation times at $\mathrm{pH}$ 3.0. Average values and error bars are deducted from three independently performed cultivations and stability measurements. PaHNL5-L1Q corresponds to the wildtype PaHNL5.

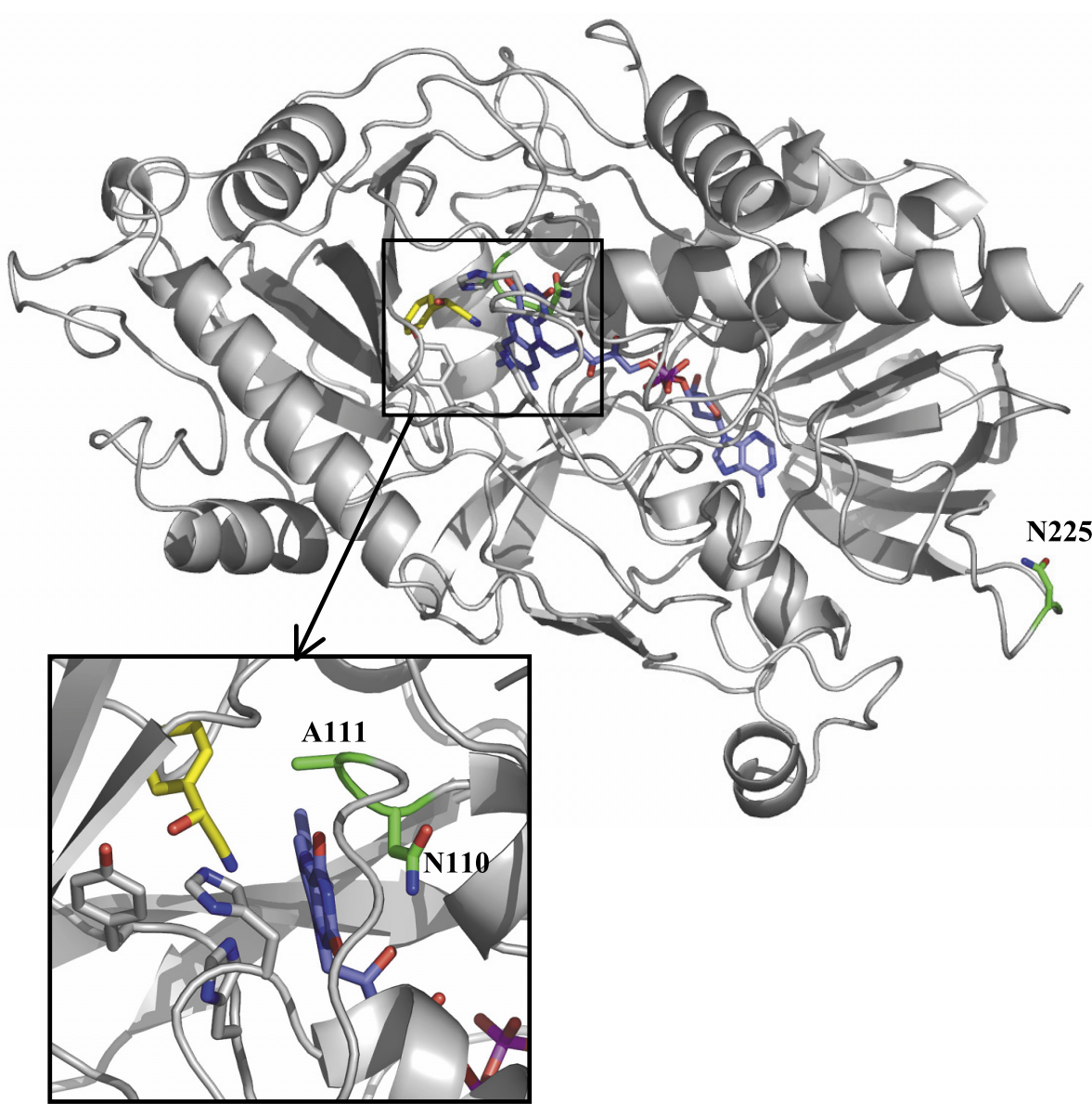

Fig. 3. Modelled enzyme substrate complex of PaHNL5 [55] indicating the main mutations described in the text: asparagine 225 (N225) at the periphery of the enzyme, and asparagine 110 (N110) and alanine 111 (A111) located at the active site (mutated sites - green, isoalloxazine portion of the FAD cofactor - blue, mandelonitrile - yellow, and the catalytic site with tyrosine 458 , histidine 460 and histidine 498 - greyish). results and to exclude structural side-effects of the serine residue at this specific position in the protein, asparagine 118 was changed to the structurally closer related glutamate (N118D). However, mutation N118D led to even faster inactivation than N118S: after 8 min incubation at $\mathrm{pH} 3$ no residual activity was detectable (Fig. 2). PaHNL5 was also subjected to digestion by endoglycosidase $\mathrm{H}$ (EndoH) that specifically cleaves the attached sugar chains from glycosylated sites, but leaves one GlcNAc attached to the corresponding asparagine. This 'truncated' variant showed only slightly decreased stability at low $\mathrm{pH}$ (Fig. 2) compared to fully glycosylated $\mathrm{PaHNL} 5$ which indicates that either just the core glycosylation at position N118 is sufficient for stability at low pH or that the glycosylic moiety at N118 is not accessible for EndoH treatment. However, the glycosylation site N118 was shown to be essential for the stability of PaHNL5 at low $\mathrm{pH}$.

The investigation of the functionality of N-glycosylation sites of PaHNL5 using serine scanning yielded a mutein (N225S) exhibiting improved catalytic properties. This glyco-variant catalyzed the stereospecific addition of $\mathrm{HCN}$ to 3-phenylpropionaldehyde with a higher conversion rate and improved enantiomeric excess than the wildtype enzyme containing all putative glycosylation sites [51]. The resulting $(R)$ cyanohydrin is an important chiral precursor for the synthesis of 'prils'. These pharmacologically active compounds belong to the class of angiotensin-converting enzyme inhibitors (ACEi) and have a wide range of applications in the treatment of hypertension and congestive heart failure [53]. Regarding the modelled structure of PaHNL5 [54] it was surprising that the glycosylation site N225 which is located in a turn linking two antiparallel beta-strands at the periphery of the enzyme (Fig. 3, [55]) has such an impact on the enzyme's catalytic properties.

\subsection{Deamidation}

Mutation A111G was introduced into $P a$ HNL5 to increase the enzymatic activity for substituted benzaldehydes. This mutein showed a 6-fold increase in specific activity for the conversion of 2-chlorobenzaldehyde to the industrially important chiral intermediate 2-chlorobenzaldehyde cyanohydrin [48][52]. However, expression of the recombinant protein suffered from decreased enzyme productivity compared to the unchanged wildtype protein. Analysis of PaHNL5-A111G by LC/MS/ MS revealed that in addition to the existing mutation at position 111, where a glycine residue was substituted for alanine, the adjacent position 110 showed an aspartate instead of the expected asparagine. Due to the exchange of alanine at position 111, the 
neighbouring residue N110 was subjected to posttranslational deamidation. Since the polypeptide analysis of the unchanged wildtype protein resulted in the regular sequence N110-A111, the newly introduced glycine residue can be made responsible for the observed posttranslational deamidation. Consequently, the question arose which of the two mutations was truly accountable for the enhanced properties of the enzyme. The double mutein N110DA111G anticipated the deamidation event and led to about $20 \%$ higher productivity, reaching wildtype levels. Most important, both the high level of conversion and the high enantiomeric excess (ee) using the substrate 2-chlorobenzaldehyde were maintained. The mutation N110D alone was not competitive in the synthesis reaction leading to 2-chloromandelonitrile. Thus, it could be confirmed that just the mutation A111G was responsible for the enhanced synthesis properties, whereas anticipation of the posttranslational deamidation bypassed the bottleneck in enzyme production [40]. In general, this strategy could be applied to prevent limitations caused by already existing or newly created posttranslational modifications, relieving some metabolic burden from the cells.

\section{Conclusion}

Posttranslational modifications such as glycosylation, sulfation, and deamidation or in vitro manipulation strategies like PEGylation provide interesting targets for engineering purposes not only for biomedical applications, but also, increasingly, for useful biocatalysts. The necessity to work under reaction conditions that are mostly unnatural and unfavourable for native enzymes leads to an increasing demand for robust engineered enzymes. Influencing posttranslational modifications could limit or even overcome disadvantageous deficiencies of enzymes as catalysts and recently demonstrated great advantages for the future of enzyme engineering.

\section{Acknowledgements}

We thank DSM, the FFG, the Province of Styria, SFG and the City of Graz for financial support.

\section{Received: July 12, 2005}

[1] V. Gomord, L. Faye, Curr. Opin. Plant Biol. 2004, 7, 171.

[2] S.R. Hamilton, P. Bobrowicz, B. Bobrowicz, R.C. Davidson, H. Li, T. Mitchell, J.H. Nett, S. Rausch, T.A. Stadheim, H. Wischnewski, S. Wildt, T.U. Gerngross, Science 2003, 301, 1244.

[3] A.M. Aly, M. Higuchi, C.K. Kasper, H.H. Kazazian, A.E. Antonarakis, L.W. Hoyer, Proc. Natl. Acad. Sci. USA 1992, 89, 4933.
[4] H. Lubon, W.N. Drohan, R.K. Paleyanda (American Nat. Red Cross, US), US 5965789, 1999.

[5] J. Hollister, E. Grabenhorst, M. Nimtz, H Conradt, D.L. Jarvis, Biochemistry 2002, 41, 15093.

[6] C.Y. Choi, S.H. Kang, T.J. Kang, J.H Woo, S.K. Lee, S.W. Cho (Dreambiogen Co., Ltd., S. Korea), US 2002106719-A1, 2002.

[7] H. Lis, N. Sharon, Eur. J. Biochem. 1993 $218,1$.

[8] R.J. Woods, C.J. Edge, R.A. Dwek, Nat Struct. Mol. Biol. 1994, 1, 499.

[9] L. Kasturi, J.R. Eshleman, W.H. Wunner, S.H. Shakin-Eshleman, J. Biol. Chem. 1995, 270, 14756.

[10] S.H. Shakin-Eshleman, S.L. Spitalnik, L. Kasturi, J. Biol. Chem. 1996, 271, 6363.

[11] S.E. O'Connor, B. Imperiali, Chem. Biol. 1996, 3, 803.

[12] A. Kato, S. Nakamura, M. Ban, H. Azakami, K. Yutani, Biochim. Biophys. Acta 2000, 1481,88 .

[13] C. Wang, M. Eufemi, C. Turano, A. Giartosio, Biochemistry 1996, 35, 7299.

[14] R. Kundra, S. Kornfeld, J. Biol. Chem. 1999, 274, 31039.

[15] P.M. Rudd, H.C. Joao, E. Coghill, P. Fiten, M.R. Saunders, G. Opdenakker, R.A. Dwek, Biochemistry 1994, 33, 17.

[16] U. Stochaj, M. Cramer, H.G. Mannherz, Biochim. Biophys. Acta 1992, 1122, 327.

[17] R.H. Khan, S. Rasheedi, S.K. Haq, J. Biosci. 2003, 28, 709 .

[18] B. Imperiali, K.W. Rickert, Proc. Natl. Acad. Sci. USA 1995, 92, 97.

[19] S. Hemmerich, D. Verdugo, V.L. Rath, Drug Discov. Today 2004, 9(22), 967.

[20] R.W.H. Lee, W.B. Huttner, J. Biol. Chem. 1983, 258, 11326

[21] P.A. Baeuerle, W.B. Huttner, J. Cell. Biol. 1987, 105, 2655.

[22] R.W.H. Lee, W.B. Huttner, Proc. Natl. Acad. Sci. USA 1985, 82, 6143.

[23] K.L. Moore, J. Biol. Chem. 2003, 278(27), 24243

[24] S.R. Stone, J. Hofsteenge, Biochemistry 1986, 25, 4622 .

[25] M. Higuchi, C. Wong, L. Kochhan, K. Olek, S. Aronis, C.K. Kasper, H.H. Jr. Kazazian, S.E. Antonarakis, Genomics 1990 , $6(1), 65$.

[26] A. Leyte, H.B. van Schijndel, C. Niehrs, W.B. Huttner, M.P. Verbeet, K. Mertens, J.A. van Mourik, J. Biol. Chem. 1991, 266, 740.

[27] M.C. Beinfeld, Life Sci. 2003, 72(7), 747.

[28] J. Cacia, C.P. Quan, M. Vasser, M.B. Sliwkowski, J. Frenz, J. Chromatogr. 1993, 634,229

[29] G.W. Becker, P.M. Tackitt, W.W. Bromer, D.S. Lefeber, R.M. Riggin, Biotechnol. Appl. Biochem. 1988, 10, 326.

[30] P. Galletti, D. Ingrosso, C. Manna, G. Clemente, V. Zappia, Biochem. J. 1995, 306 313.

[31] H.T. Wright, Protein Eng. 1991, 4, 283.
[32] A.B. Robinson, Proc. Natl. Acad. Sci. USA 1974, 71, 885

[33] D. Ingrosso, S. Clarke, Adv. Exp. Med. Biol. 1991, 307, 263.

[34] R. Paleari, E. Paglietti, A. Mosca, M. Mortarino, L. Maccioni, S. Satta, A. Cao, R Galanello, Clin. Chem. 1999, 45, 21.

[35] N. Declerck, M. Machius, G. Wiegand, R Huber, C. Gaillardin, J. Mol. Biol. 2000 , 301,1041 .

[36] A. Giordano, R. Cannio, F.L. Cara, S. Bartolucci, M. Rossi, C.A. Raia, Biochemistry 1999, 38, 3043.

[37] K.H. Oh, S.H. Nam, H.S. Kim, Prot. Eng. 2002, 15, 689 .

[38] S.J. Tomazic, A.M. Klibanov, J. Biol. Chem. 1988, 263, 3086.

[39] S.J. Tomazic, A.M. Klibanov, J. Biol. Chem. 1988, 263, 3092.

[40] R. Gaisberger, R. Weis, R. Luiten, W. Scranc, M. Wubbolts, H. Griengl, A. Glieder, submitted to J. Biot.

[41] J.M. Harris, R.B. Chess, Nat. Rev. Drug. Discov. 2003, 2(3), 214

[42] P. Bailon, W. Berthold, Pharm. Sci. Technol. Today 1998, 1, 352.

[43] S. Zaplinsky, C. Lee, in 'Polyethylene Glycol Chemistry: Biotechnical and Biomedical Applications', Ed. J.M. Harris, Plenum Press, New York, 1992, p. 347.

[44] R.J. Goodson, N.V. Katre, Biotechnology 1990, 8, 343.

[45] A. Kozlowski, J.M. Harris, J. Control. Release. 2001, 72(1-3), 217.

[46] C. Monfardini, O. Schiavon, P. Caliceti, M. Morpurgo, J.M. Harris, R.M. Veronese, Bioconjug. Chem. 1995, 6(1), 62.

[47] Y. Inada, M. Furukawa, H. Sasaki, Y. Kodera, M. M. Hiroto, H. Nishimura, A. Matsushima, Trends Biotechnol. 1995, 13(3), 86.

[48] A. Glieder, R. Weis, W. Skranc, P. Poechlauer, I. Dreveny, S. Majer, M. Wubbolts, H. Schwab, K. Gruber, Angew. Chem. Int Ed. 2003, 42, 4815.

[49] F. Effenberger, S. Forster, H. Wajant, Curr Opin. Biotechnol. 2000, 11, 532.

[50] H. Groeger, Adv. Synth. Catal. 2001, 343 , 547.

[51] R. Weis, R. Gaisberger, K. Gruber, A Glieder, submitted to J. Biot.

[52] R. Weis, P. Poechlauer, R. Bona, W. Skranc, R. Luiten, M. Wubbolts, H. Schwab, A. Glieder, J. Mol. Catal. B: Enzym. 2004, 29, 211.

[53] R.K. Tikare, WO2002042244, 2004

[54] I. Dreveny, K. Gruber, A. Glieder, A Thompson, C. Kratky, Structure (Camb.) 2001, 9, 803.

[55] I. Dreveny, C. Kratky, K. Gruber, Protein Sci. 2002, 11(2), 292. 